\title{
Response to tozinameran (BNT162b2) booster in twice-vaccinated kidney transplant and maintenance dialysis patients
}

\author{
Iddo Z. Ben-Dov ${ }^{1}\left(\mathbb{D} \cdot\right.$ Keren Tzukert $^{1} \cdot$ Michal Aharon $^{1} \cdot$ Hadas Pri-Chen $^{1} \cdot$ Yonatan Oster $^{2} \cdot$ Esther Oiknine-Djian $^{2}$. \\ Dana G. Wolf ${ }^{2} \cdot$ Michal Dranitzki Elhalel $^{1}$
}

Received: 4 November 2021 / Accepted: 16 December 2021 / Published online: 17 January 2022

(c) The Author(s) under exclusive licence to Italian Society of Nephrology 2022

Keywords COVID-19 · Viral infections · Booster $\cdot$ Vaccines $\cdot$ End stage renal disease $\cdot$ Kidney transplantation $\cdot$ Hemodialysis · Peritoneal dialysis

On 22-Sep-2021 the U.S. Food and Drug Administration amended the emergency use authorization for the PfizerBioNTech COVID-19 Vaccine (tozinameran) to allow the use of a single booster dose, to be administered at least 6 months after completion of the primary series in certain populations including individuals 18 years of age and older at high risk of severe COVID-19 [1], while authorization for immunosuppressed patients was given 1 month earlier. However, boost vaccination of solid organ recipients commenced in Israel as early as mid-July. Additional at-risk populations, including elderly individuals, patients treated with maintenance dialysis and healthcare workers trailed briefly; the general adult population soon followed, resulting in increased protection from infection. Patients needing chronic renal replacement therapy are at risk for severe COVID-19 and have been shown to mount a lesser humoral response to mRNA vaccination [2]. In our single-center cohort we observed that reduced vaccine-elicited antibody levels in dialysis and kidney transplant patients were associated with increased infection rates [3]. Herein, we describe the short-term impact of booster administration in these patients, amidst a third wave of infections and deaths currently experienced in Israel primarily by

Iddo Z. Ben-Dov

iddo@hadassah.org.il

1 Department of Nephrology and Hypertension,

Hadassah Medical Center and the Faculty of Medicine,

Hebrew University of Jerusalem, Kiryat Hadassah 1,

921120 Jerusalem, Israel

2 Department of Clinical Microbiology and Infectious Diseases, Hadassah Medical Center and the Faculty of Medicine, Hebrew University of Jerusalem, Kiryat Hadassah 1, 921120 Jerusalem, Israel the Delta variant, possibly amplified by waning immunity (Online Resource 1).

Prior to initiation of vaccination we launched the COVID19 mRNA Vaccine Immunogenicity in patients with end stage Renal Disease (COVIReD) prospective cohort study designed to investigate the long-term kinetics and implications of antibody response to COVID-19 vaccine and infection in this vulnerable population. The study was approved by the local ethical Committee and its methods and preliminary results have been recently published [3]. In this update, we report patients' humoral responses to booster vaccination. Antibodies were quantified using the LIAISON SARS-CoV-2 S1/S2 IgG test (DiaSorin, Sallugia, Italy). Risk factors for low antibody levels were assessed using a generalized linear mixed effects model accounting for repeated measurements (lme4/R statistical package). Infection risk was modeled with logistic regression.

In total, we quantified anti-S1/S2 IgG levels at multiple time points in 183 maintenance dialysis patients, 280 kidney transplants and 74 controls not previously infected with COVID-19, of whom 82, 118 and 23, respectively, provided samples after receiving a vaccine booster. Figure 1a presents antibody median values across study groups and vaccination episodes.

We had previously noted that with a most recent antibody level at or above $59 \mathrm{AU} / \mathrm{ml}$ no post-vaccination participant became infected, suggesting a COVID-19 protective cutoff [3]. Figures $1 \mathrm{~b}$ and $\mathrm{c}$ show that prior to boosting (pre.v3), this level was achieved by $76 \%$ of controls, $35 \%$ of dialysis patients and $12 \%$ of transplant patients, while 8-117 days after booster injection respective rates were $100 \%, 91 \%$ and $56 \%$. Risk factors for antibody levels $<59$ AU/ml despite booster injection (control subjects excluded) were being in the transplant group, $\mathrm{OR}=15.0$ vs. dialysis 


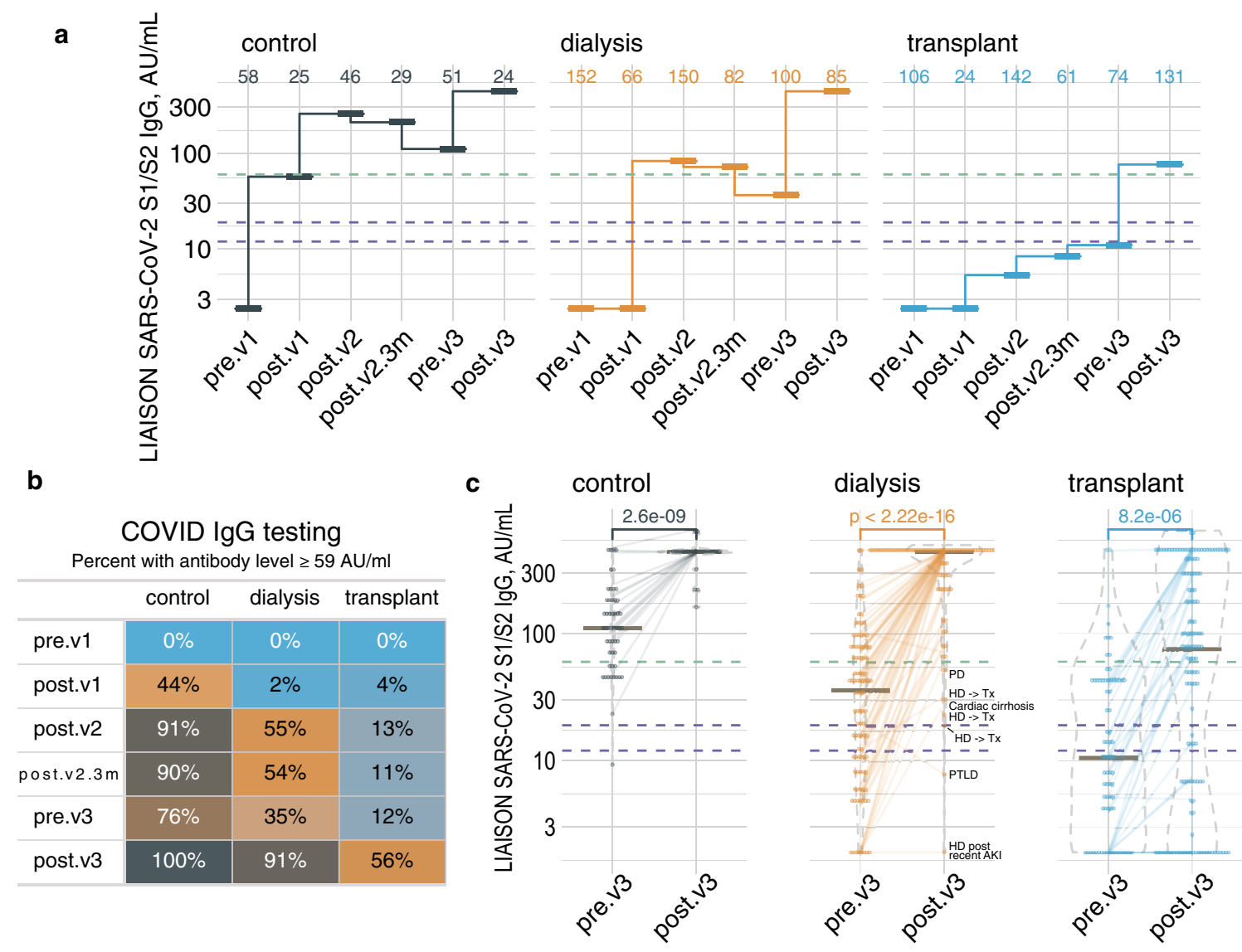

Fig. 1 Antibody levels repeatedly measured across study groups and time points in patients not previously infected with COVID-19. a Median plots showing the summarized antibody level in a logarithmic scale in controls (left), dialysis patients (middle) and transplant patients (right). The dashed purple lines represent the equivocal range (12-19 AU/ml), while the dashed green line represents our proposed protective threshold, $59 \mathrm{AU} / \mathrm{ml}$. b The percentage of participants with threshold or higher antibody level at each time point, by group. c Dot and violin plots, with horizontal lines at medians, focused on the latest study time points. Semi-transparent lines connect repeated measurements from the same participant. Time point abbreviations: pre.v1, before vaccination; post.v1, after a single jab; post.v2, up to 10 weeks after the second dose; post.v2.3m, 3-5 months after double vaccination; pre.v3, 6 months after double vaccination; post.v3, 8-117 days after the booster shot (colour figure online)

of high-risk chronic renal replacement therapy patients, as it did in healthcare controls. While the association between achieved binding antibody titers and resistance to viral infection is an open question, most patients have now reached a level likely to afford protection from disease, and specifically severe disease. Interestingly, the anti SARS-CoV-2 IgG levels were higher after the third dose than after the previous doses which may hint that the latest immune response may be more robust and hopefully sustainable, even in immunecompromised patients. In fact, kidney transplant recipients showed the most striking enhancement in humoral response to the booster dose, exhibiting an almost sixfold increase in the number of patients developing a protective level of antibodies. This is greater than previously described [4], and supports repeated vaccination of non-responsive immunosuppressed patients. However, many transplant patients remain below threshold in terms of antibody levels even 


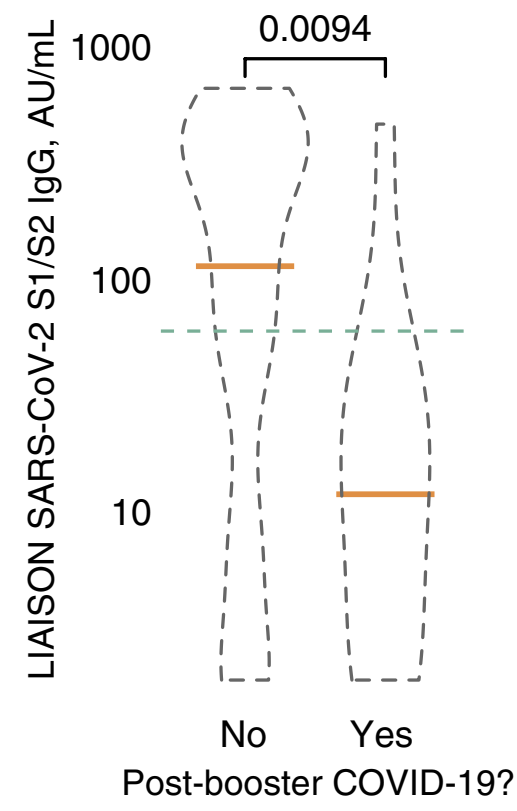

Fig. 2 Anti SARS-CoV-2 S1/S2 IgG levels in patients and controls who experienced COVID-19 infection after receiving a booster dose of the BNT162b2 vaccine (right violin plot, $n=19$ ) versus those who were not infected (left violin plot, $n=479$ ). The green dashed line represents the proposed $59 \mathrm{AU} / \mathrm{ml}$ protective cutoff. Statistical significance was not affected by adjustment for age, sex or study group (not shown) (colour figure online)

after boosting. In our transplant clinic we did not lower immunosuppression prior to the booster dose, although various transplant centers contemplated transiently reducing immunosuppression to facilitate response [5]. Our experience is consistent with "real life" practice and may be of use to guide practical recommendations for kidney patients receiving immunosuppression or dialysis.

Supplementary Information The online version contains supplementary material available at https://doi.org/10.1007/s40620-021-01235-3.

Author contributions Study conception and design-IZB-D, DGW, MDE. Data acquisition including patient recruitment-MA, KT, HP-C,
EOD. Data analysis-IZB-D. Data interpretation-IZB-D, KT, HP-C, YO, MDE. Drafting the manuscript-IZB-D, KT, HP-C, YO, MDE. Revising the manuscript-IZB-D, KT, HP-C, YO, EOD, DGW, MDE.

Funding There was no external funding for this study. However, the Ministry of Health of the State of Israel supported this study by supplying serology kits to the hospital's virology laboratory.

\section{Declarations}

Conflict of interest On behalf of all authors, the corresponding author states that there is no conflict of interest.

\section{References}

1. @US_FDA (2021) FDA authorizes booster dose of Pfizer-BioNTech COVID-19 vaccine for certain populations. FDA. Wed, 09/22/2021-19/27/2021

2. Hou Y, Lu K, Kuo K (2021) The efficacy of COVID-19 vaccines in chronic kidney disease and kidney transplantation patients: a narrative review. Vaccines 9(8):885. https://doi.org/10.3390/vacci nes9080885

3. Ben-Dov IZ, Oster Y, Tzukert K et al (2021) Impact of tozinameran (BNT162b2) mRNA vaccine on kidney transplant and chronic dialysis patients: 3-5 months followup. J Nephrol https:// doi.org/10.1101/2021.06.12.21258813

4. Werbel W, Boyarsky B, Ou M et al (2021) Safety and immunogenicity of a third dose of SARS-CoV-2 vaccine in solid organ transplant recipients: a case series. Ann Intern Med 174(9):1330. https://doi.org/10.7326/L21-0282

5. Yahav D, Rozen-Zvi B, Mashraki T et al (2021) Immunosuppression reduction when administering a booster dose of the BNT162b2 mRNA SARS-CoV-2 vaccine in kidney transplant recipients without adequate humoral response following two vaccine doses: protocol for a randomised controlled trial (BECAME study). BMJ Open 11(10):e055611. https://doi.org/10.1136/bmjop en-2021-055611

Publisher's Note Springer Nature remains neutral with regard to jurisdictional claims in published maps and institutional affiliations. 